\title{
Analysis of Amplify Forward, Decode and Amplify Forward, and Compression Forward Relay for Single and Multi-node Cognitive Radio Networks
}

\author{
B.Perumal ${ }^{1}$, J.Deny2, R. Raja Sudharsan ${ }^{3}$, E. Muthukumaran ${ }^{4}$, R. Raja Subramanian ${ }^{5}$ \\ \{ palanimet@gmail.com ${ }^{1}$, prof.j.deny@gmail.com², rajasudharsan5255@gmail.com³, \\ reachmkumaran@gmail.com ${ }^{4}$, rajasubramanian.r@klu.ac.in ${ }^{5}$ \} \\ Department of Electronics and Communication Engineering, Kalasalingam Academy of Research \\ and Education, Krishnan Koil, Virudhunagar, 626126, India. ${ }^{1,2,3}$ \\ Department of Electronics and Communication Engineering, Dr. B. R. Ambedkar Institute of \\ Technology, Andaman and Nicobar Islands, India ${ }^{4}$ \\ Department of Computer Science Engineering, Kalasalingam Academy of Research and Education, \\ Krishnan Koil, Virudhunagar, 626126, India ${ }^{5}$
}

\begin{abstract}
Intellectual radio is a developing remote innovation utilized to expand the recurrence range usage of the constrained ghastly assets. Helpful interchanges have been considered as a potential strategy for comprehending the blurring impacts, for example, improving the steadfastness of system execution, arrange throughput, and versatility in remote correspondences. The exhibitions of Single node -AF (Amplify and Forward) and multiple nodes - DAF (Decode, Amplify and Forward) intellectual hand-off systems are examined over autonomous non indistinguishably circulated Weibull blurring diverts in Cognitive Radio Network. The diagnostic outcomes show that the bit mistake rate and the Signal to Noise Ratio esteem is $24 \mathrm{~dB}$ for Single and Multiple nodes in network are better than handing-off conventions.
\end{abstract}

Keywords: Amplify Forward, Decode and Amplify Forward, Compression Forward, Single and Multi-node, Cognitive radio networks

\section{Introduction}

Radio range is a costly help in remote correspondence. In any case, it has been demonstrated that the vast majority of the ranges are underutilized in huge geographic districts of the world. This underutilization has brought about the availability of range band named as range gaps. The intellectual radio system is the main way out for this unearthly wastefulness. The subjective radio is one of the most amazing methods that empower to misuse the range gaps without influencing the essential client. A framework, that "detects its operational electromagnetic condition can powerfully and self-governing change its radio working parameters to alter the framework operation's, for example, augmenting throughput, moderating impedance, encouraging interoperability, getting to auxiliary markets". The essential capacity of the subjective radio is to permit the optional client to use without influencing the essential client.

The significant test of the CR systems is to full-fill the nature of administration which is the need of the PUs and it augments the throughput of the SUs. The separation between the transmitter and the collector is exceptionally huge and it causes multipath blurring, shadowing and way misfortune. Helpful range detecting will viably handle the above difficulties. Range detecting has been utilized to recognize the range gaps (blank areas) in the radio Spectrum and 
it is designated to Secondary User (Unlicensed User or Cognitive User) without influencing the essential client (Licensed User). Detecting can be performed by Cooperative range detecting and non-agreeable range detecting.

Helpful range detecting shares detecting data in order to get progressively effective outcome and it has brought together, dispersed and transfer helped approach. At the point when the immediate connection isn't offered between two hubs or when the nature of the channel between these hubs is poor, transferring plan is frequently acquainted with line up a dependable correspondence between 2 hubs. Hand-off hubs are put between the source and the goal so it turns out to be simple for the recipient to evaluate the first sign. There are not many transferring conventions that help in improving the sign quality for example, AF, DF, DAF and CF. Helpful detecting is utilized to expand the spatial assorted variety and limit. In this paper, both single jump and multi bounce transferring conventions have been broke down by utilizing Signal to clamor proportion, Signal to bit blunder rate and furthermore throughput of the system. The reproduction results show that the presentation of multi-bounce is better than the single jump transferring convention.

The remnants of this article have been organized with different areas as follows by introduction: related works are introduced in Segment II. Dissect of Cooperative correspondence over single jump and multi bounce systems are introduced in Segment III. Portrayal of the framework model is delineated in Segment IV. Simulation results are examined in Section V. Segment VI finishes with conclusion.

\section{Related Works}

Propelled by the fundamental strategies of the old-style AF, translate and-forward and pack and-forward handing-off plans have been applied to psychological radio. In [1] Hybrid translate enhance forward steady helpful decent variety convention has been inferred utilizing SNR-based transfer choice. Shut structure articulations for the PDF, blackout likelihood and the normal divert limit in regular helpful correspondence with AF hand-off system over Rayleigh blurring channel were inferred utilizing MRC strategy in [2].

In [3], shut structure articulations of blackout likelihood and ergodic limit of a two way intensify and forward transfer channel with Rayleigh blurring were inferred. As of late, Cooperative handing-off strategies have picked up consideration as a productive method to relieve blurring in remote systems [4]. The vast majority of the traditional agreeable transfer systems are Half-Duplex (HD) and they utilize transferring methods [5]. In [6] two-way AF and DF helpful transferring conventions have been acquainted with improve the ghostly effectiveness.

The otherworldly productivity misfortune the normal aggregate pace of two-way AF HD transferring tight upper and lower limits are determined [7]. Start to finish execution of intensify and forward double bounce agreeable assorted variety arrange the summed-up choice plan over free Rayleigh blurring diverts is utilized. In [7] Outage likelihood of a two-way intellectual transfer arrange has been determined utilizing unravel and forward (DF) convention over Rayleigh blurring channels. The blackout likelihood of a multi-AF-hand-off framework with the Best Relay Selection (BRS) has been broke down in.

The Weibull appropriation assumes a significant job in a few logical fields, for example, in radar mess, dependability building, and disappointment information examination. The Weibull model shows a great fit to exploratory blurring channel estimations, for both indoor and open-air situations. A physical avocation for displaying remote blurring channels with the Weibull dissemination. 
Numerous specialists have worked dependent on agreeable range detecting framework over Rayleigh blurring and Nagakami blurring channels and they have been researched for AF and DF. Image mistake likelihood of DF over Rayleigh blurring channels has been investigated. So also, Relaying systems are breaking down over free indistinguishably circulated for Weibull blurring channels. Blackout execution of double bounce AF transfer has been examined over Nagakami blurring channels researched. It is obvious from the writing audit that the vast majority of the works depend on AF or DF singlejump convention. Henceforth, AF, DAF and CF have been considered in the current work. Further the presentation of single-jump and multi-bounce handing-off plans with Weibull blurring channels are broke down. By utilizing the multi jump transferring plan the effectiveness of range usage can be expanded, the obstruction among clients can be decreased, increasingly over the system throughput and the administration inclusion territory can be expanded.

\section{Analytical representations of Networks}

A system which comprises of one root node (R) and one terminus node (T) has been associated straightforwardly and furthermore by means of AF. Henceforth, known as single bounce transferring plan and illustrated in fig. 1 .

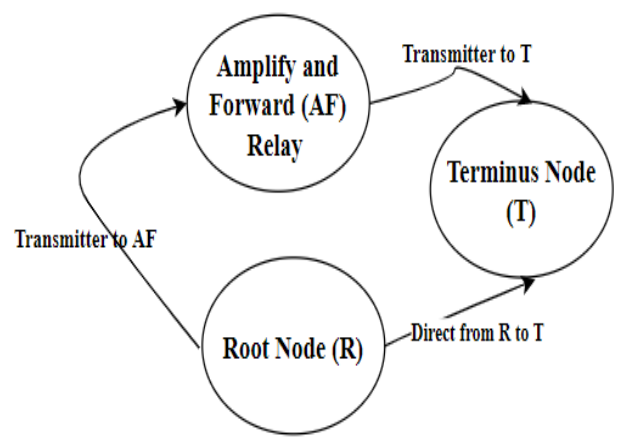

Fig 1. Single node Amplify and Forward Relay (Half- Duplex)

This situation is undifferentiated from a downlink framework in a one-end communication system. The information is sent from root node to terminus node through direct connection and afterward, the incoming signal is given to terminus node $(\mathrm{T})$,

$$
Z_{R T}=y_{v}(x) g_{R T} J_{v}+v(1)
$$

Where, $y_{V}(x)$ denotes incoming signal that is detected signal from the network, $g_{R T}$ represents blurring channel coefficient between the root and the terminus node and $l_{v}$ means the transmitted force.

$$
l_{R}+J_{T}=J_{v}
$$

This hand-off is outfitted with two receiving wires, one is for broadcast and another is for gathering. Transmitter is utilized to transmit the signals from the hand-off to terminus node and Terminus node as receiver is utilized to get the signal from root node. The source needs to transmit the detecting sign to the goal and it has two stages. In the primary stage, $y_{D}(x)$ the root node imparts signal to transfer with blurring coefficient $g_{R T}$. The got signal at the AF hand-off is

$$
Z_{R v}=y_{v}(x) g_{R r} J_{R}+v_{s}
$$


Where, $g_{R T}$ represents incoming signal, $v_{g}$ denotes added substance whit Gaussian commotion with mean zero and the Variance at the AF hand-off. is network blurring coefficient between root node and transfer and it has been determined by utilizing sign to clamor proportion of root node-to-hand-off connection $Z_{R Y}$.

$$
Z_{R Y}=\mid g_{R Y} \|^{2} J_{R} / P_{\text {noise }}
$$

In the subsequent stage, the incoming signals from Amplify and Forward signal relay hand-off are enhanced with amplification factor $\eta$ and re-transmitted to the terminus node. The incoming signal at the terminus node given by,

$$
K_{S T}=\eta g_{S T} Z_{R T} J_{T}+v_{T}(5)
$$

Here, $K_{S T}$ denotes incoming signal from the transfer to terminus node. $l_{R}$ and $l_{T}$ represents transmitted force at the root and terminus node, separately. $v_{T}$ is the added substance white Gaussian clamor with mean noise and change at the terminus node $(\mathrm{T})$

$$
\begin{gathered}
\eta=\sqrt{J_{V / g_{R T} J_{R}+\mu^{2}}}(6) \\
\eta=\sqrt{J_{V / g_{R T} J_{R}+0.1}}(7)
\end{gathered}
$$

Where, $\eta$ denotes the amplification factor of Amplify and forward relay.

$$
Z_{R T}=J_{R} J_{T}\left|g_{S T}\right|^{2}\left|g_{R T}\right|^{2} / 0.1+J_{R}\left|g_{S R}\right|^{2}+J_{V}\left|g_{R T}\right|^{2}
$$

The above Equ. (8) depicts that analytical representation of single node AF relay.

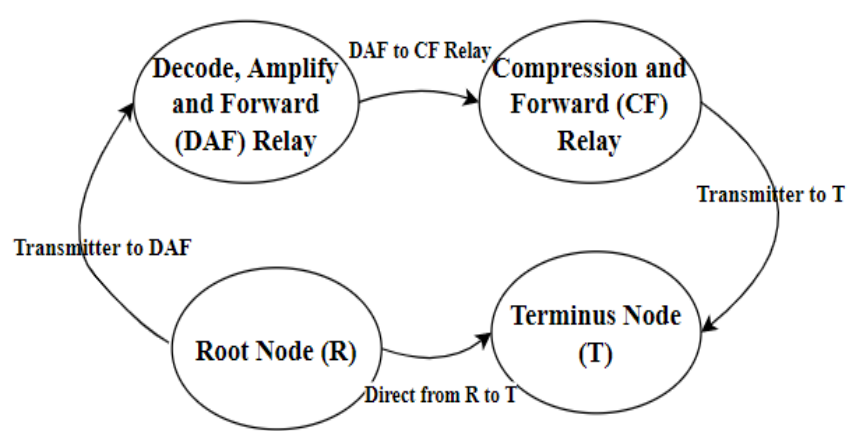

Fig 2.Multiple nodes Decode, Amplify and Forward Relay and Compression \& Forward Relay (HalfDuplex).

In fig 2, the system comprises of one root node $(\mathrm{R})$ and one terminus node $(T)$ with at least two relays and thus, it is called multi bounce handing-off plan. In this plan, the DAF is the blend of decipher advance and Amplify and Forward, and CF transfer. This situation is additionally similar to downlink framework in HD framework. The root node needs to convey signal $y_{v}$ ( to terminus node. This hand-off will translate the message completely. This procedure requests part of registering period. Though, it has a preferred position that a mistake amending code likely could be handled inside the hand-off. In the event that the transfer station doesn't have PC control to absolutely interpret the message, the approaching sign will basically be decoded and re-encoded without image by image. Along these lines, there's no 
enhanced clamor in the imparted sign. At that point the sign is intensified and sent to back to back hand-off. During this case, there are 3 phases.

In primary phase, root node sends information to hand-off DAF, the blend of Decode and Forward, and Amplify and forward. The signal $y_{v}$ (is first decoded and afterward the signal is amplified. In secondary phase, the resultant signal is re-communicate prompted additional hand-off known as Compression Forward. The tertiary phase, the packed signal sent to terminus node. In the hand-off, Decode, Amplify and Forward has the benefits of both Amplify and Forward, and Decode and Forward. The incoming signals at first hand-off or relay.

$$
Z_{R T(\text { multi-nodes })}=\sqrt{ }\left(J_{R} / J_{T}\right) y_{V}(x)_{\text {multi-nodes }} * g_{R T}(9)
$$

Where, $y_{v}(x)_{\text {multi-nod }}$ represents the Decode and amplify forward signal of $y_{v}$ (coefficient. The DAF signal of multi-nodes can be given by,

$$
Z_{R T(m u l t i-n o d e s)}=Z_{R T(m u l t i-n o d e s)} \text { (10) }
$$

Where $y_{v}(x)_{\text {multi-nod }}$ represents sign transmitted from the source to the transfer. has been characterized in the above condition and $\eta$ denotes enhancement factor. After Decode and Amplify Forward, the sign is retransmit driven the following hand-off i.e., CF convention. In this CF for transfer systems is limit achieving, the sign pressure is made by the math coding. Number-crunching coding is a typical calculation utilized in both lossless and lossy information pressure algorithms. It is an entropy encoding strategy, in which much of the time seen images are encoded with less bits than the seldom observed images. The advantages are: of this coding are the pressure proportion is higher contrasted with Huffman coding, productivity is more noteworthy and excess is abundantly decreased. The compacted signal is sent to the goal. The got signal at the goal is numerically given as

$$
Z_{S_{\text {en } T}}=\operatorname{compr}\left(Z_{\text {RT } \text { multi-nodes }}\right.
$$

A data has been transmitted from root node to terminus node through Weibull blurring channel. Here Weibull blurring channel is utilized because it is straightforward and a versatile model, which effectively suits with the trial estimations for indoor and open-air conditions. Additionally, it considers the separation amid root node and terminus node has equally considered as excessively enormous, which causes blurring. To diminish the blurring impacts, present hand-off helped agreeable range detecting. In this framework hand-off is put among source and goal. From the source to goal, there are two connections, right off the bat the immediate connection between the source and the destination, and another connection through hand-off, and this might be single transfer or various hand-off.

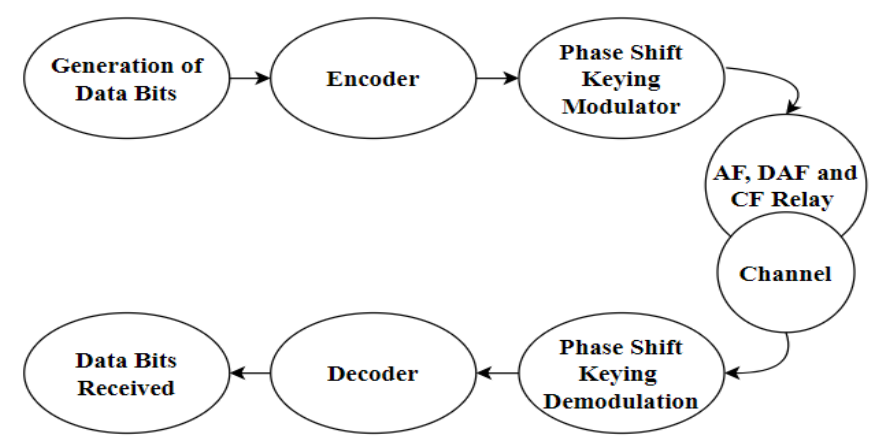

Fig 3.Flow of the Relay System 
In fig. 3. Block graph comprises of three squares in particular Transmitter, hand-off and Receiver. At the transmitter initial step is to produce arbitrary numbers which is appeared in Generation of bits and the advanced information are changed over in to simple information, at that point the information is encoded by utilizing number-crunching encoding process, at that point the information is balanced by PSK adjustment conspire. Phase shift keying (PSK) is the least complex type of PSK utilizes 2 stages which are separated by 140 degrees. In Phase shift keying information rate is low since it transmits just the slightest bit per image. In this plan we can utilize MIMO, and thus different duplicates of information can be transmitted. There are two ways in which the information is sent to terminus node. The range opening recognized by the vitality discovery strategy, contrasted with other detecting methods vitality identifier is anything but difficult to execute and furthermore without having the information on essential client. The information is gotten at the collector end by means of direct way and through transfer. Accordingly, different duplicates of information are gotten by the receiver. MRC technique is utilized to get right information. Here, SNR is determined for all the duplicates where one is greatest Signal to Noise Ratio that is the right information and it is sent to the collector. At that point the information is sent to the terminus node after the demodulation and decoding stages.

\section{Simulation Results}

From the above conversation the exhibition of single bounce and multi jump conventions are examined. The outcomes are the examination of single bounce (Amplify Forward) and multi jump (Decode and Amplify Forward and Compress Forward).

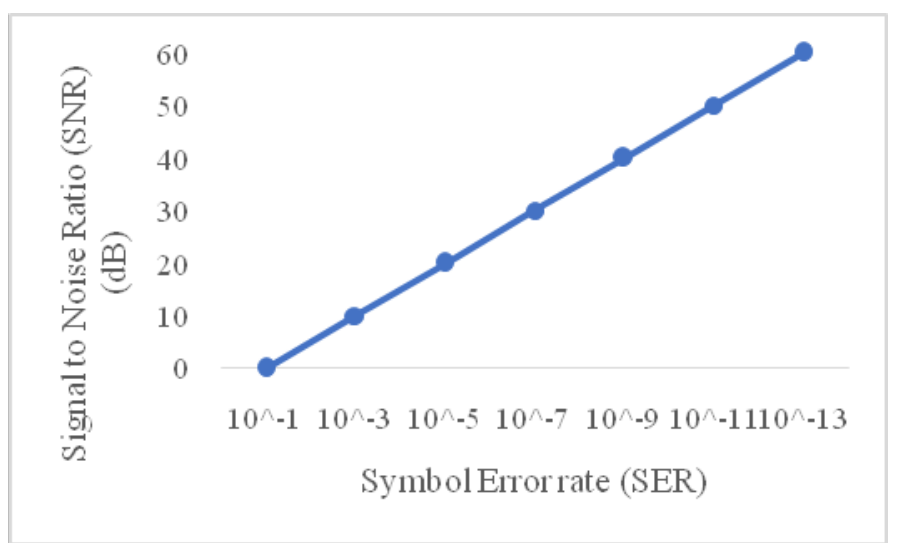

Fig 4.Computation of SNR Vs SER for Amplify and Forward Relay of Single node

The Fig. 4. portrays single bounce organize (AF). In a solitary bounce handing-off convention just one hand-off is utilized. Sign to commotion proportion of the single jump is around $27 \mathrm{~dB}$ is better than [1]. The separation between the source what's more, the terminus node is put at equivalent separation. Fig. 5(a) and Fig. 5(b) shows that the exhibition of Decode and Amplify Forward and Compress Forward transferring conventions. 


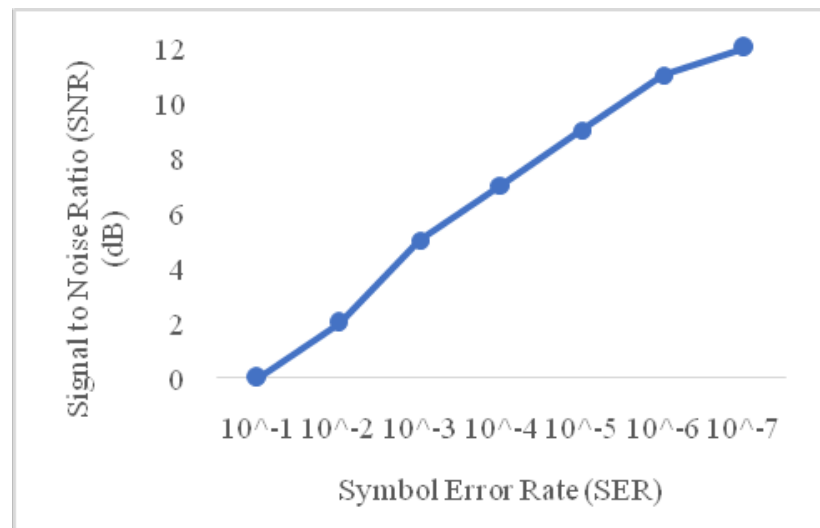

Fig 5 (a). Computation of SNR Vs SER for Decode, Amplify and Forward Relay of Multiple nodes in a network

The Fig. 5(a) and 5(b) is the correlation among AF and DAF (mix of DF and AF). The estimation of Signal to Noise Ratio is likewise shifted. Looking at two Fig. 4 and 5(a) \& 5(b) multi bounce transferring convention execution is better than burn jump regarding Signal to commotion proportion. Sign to clamor proportion is decreased in multi jump systems (Decode and Amplify Forward) than the single bounce organizes (Amplify Forward). A multi bounce transferring convention execution is better than scorch jump as for Signal to clamor proportion. Sign to clamor proportion is decreased in multi bounce systems (Decode and Amplify Forward and Compress Forward) than the single jump arranges (Amplify Forward).

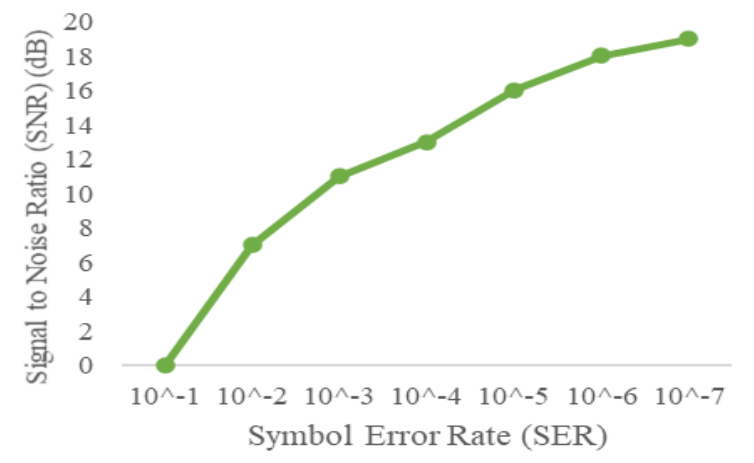

Fig 5 (b).Computation of SNR Vs SER for Decode, Amplify and Forward (DAF) \& Compression and Forward (CF) Relay of Multiple nodes in a network

The above correlation of single jump with Signal to Noise Ratio esteem is $24 \mathrm{~dB}$ which is more noteworthy than multi bounce Decode and Amplify Forward (SNR $12 \mathrm{~dB}$ ) and furthermore another multi jump DAF and CF (SNR $19 \mathrm{~dB})$.

\section{Conclusions}

When contrasted with single bounce the estimation of SNR is diminished in multi jump systems. The limit is conversely corresponding to Signal to Noise Ratio. Henceforth the limit of the system is expanded by utilizing multi bounce systems. From the above outcomes infer 
that as far as Symbol error rate and SNR, the framework utilizing multi jump Decode, Amplify Forward and Compress Forward transfers is a superior framework than the Singe bounce Amplify Forward.

\section{References}

[1] Duy, T.T. and Kong, H.Y., 2012. Performance analysis of hybrid decode-amplify-forward incremental relaying cooperative diversity protocol using SNR-based relay selection. J. Commun. Netw-S. Kor. 14, 703-709

[2] Sudharsan, R.R., Deny, J., Muthukumaran, E. et al. FPGA based peripheral myopathy monitoring using MFCV at dynamic contractions. J Ambient Intell Human Comput (2020). https://doi.org/10.1007/s12652-020-02363-7

[3] Sudharsan, RR, J. Deny, E. Muthu Kumaran, and A. Sharon Geege. "An Analysis of Different Biopotential Electrodes Used for Electromyography", 2020.

[4] Van Nguyen, B. and Kim, K., 2016. Performance analysis of amplify-and-forward systems with single relay selection in correlated environments. Sensors-Basel. 16, 1472

[5] Sudharsan, RR, and J. Deny. "Field Programmable Gate Array (FPGA)-Based Fast and Low-Pass Finite Impulse Response (FIR) Filter" In Intelligent Computing and Innovation on Data Science, pp. 199-206. Springer, Singapore, 2020.

[6] Deny, J., and Sudharsan, RR. "Block Rearrangements and TSVs for a Standard Cell 3D IC Placement." In Intelligent Computing and Innovation on Data Science, pp. 207-214. Springer, Singapore, 2020.

[7] Krishna, R. R., Kumar, P. S., \& Sudharsan, R. R. "Optimization of wire-length and block rearrangements for a modern IC placement using evolutionary techniques" In 2017 IEEE International Conference on Intelligent Techniques in Control, Optimization and Signal Processing (INCOS) (pp. 1-4). IEEE. 2017.

[8] J.Deny ,N.Sivasankari, "Biometric Security in Military Application "Journal of Engineering Procedia ELSEVIER,Vol.38,pp-1138-1144.

[9] J.Deny, Dr.M.Sundhararajan“Survey of Texture Analysis Using Histogram in Image Processing” International Journal of Applied Engineering Research (IJAER) Vol 26, pp .8737-8739.

[10] J.Deny, Dr.M.Sundhararajan "Review of Color Image Segmentation Using K-means and SuperPixel Algorithm" International Journal of Digital Communication and Networks, Vol. 2, Issue 12, pp: 4-8.

[11]Dr.E.Muthukumaran. Dr.J.Deny, Dr.B.Perumal,G. Suseendran., Dr. D.Akila "A narrative NonInvasive Diagnostic loom Based by the side of correlation of Nasal set Rhythm in addition to customary Three Radial Pulses Measurement" IOP Conf. Series: Journal of Physics: Conf. Series 1228 (2019) 012075.

[12] Deny John Samuvel, B. Perumal, Muthukumaran Elangovan, "Music recommendation system based on facial emotion recognition, Publicado en 3C Tecnología. Special Issue - March 2020. 